\title{
On the consistency of finite difference approximations of the Black-Scholes equation on nonuniform grids
}

\author{
Myles D. Baker and Daniel D. Sheng \\ (Communicated by Johnny Henderson)
}

\begin{abstract}
The Black-Scholes equation has been used for modeling option pricing extensively. When the volatility of financial markets creates irregularities, the model equation is difficult to solve numerically; for this reason nonuniform grids are often used for greater accuracy. This paper studies the numerical consistency of popular explicit, implicit and leapfrog finite difference schemes for solving the Black-Scholes equation when nonuniform meshes are utilized. Mathematical tools including Taylor expansions are used throughout our analysis. The consistency ensures the basic reliability of the finite difference schemes based on choices of temporal and variable spatial derivative approximations. Truncation error terms are derived and discussed, and numerical experiments using $\mathrm{C}, \mathrm{C}++$ and Matlab are given to illustrate our discussions. We show that, though orders of accuracy are lower compared with their peers on uniform grids, nonuniform algorithms are easy to implement and use for turbulent financial markets.
\end{abstract}

\section{An introduction of the differential equation and nonuniform grids}

Let $f=f(x, t)$ be the value of an option in the option market. In [Brandimarte 2006; Wilmott et al. 1995], for example, we find the linearized Black-Scholes equation

$$
B(f)(x, t)=\frac{\partial f}{\partial t}(x, t)+\alpha \frac{\partial^{2} f}{\partial x^{2}}(x, t)+\beta \frac{\partial f}{\partial x}(x, t)-\gamma f(x, t)=0, \quad t \geq 0,
$$

where $t$ is time, $x$ is the asset price (or space variable), and $B(f)$ is the so-called Black-Scholes operator, expressed in terms of partial derivatives. The constants

MSC2000: primary 65G50, 65L12, 65N06, 65N15; secondary 65L70, 65L80, 65D25.

Keywords: finite difference approximation, difference algorithm, explicity, implicity, consistency, accuracy, matrix equations.

This collaborated research was supported by a Undergraduate Research and Scholarly Achievement Grant (no. 223-08-URSA) from Baylor University. 
$\alpha, \beta$ and $\gamma$ are nonnegative; they represent important parameters in economic and financial calculations.

Let $T>0$ be a sufficiently large number. We consider a rectangular space-time domain $D$ where $0 \leq x \leq 1,0 \leq t \leq T$ for (1-1). We further adopt an initial option value distribution

$$
f(x, 0)=\sin (a \pi x), \quad 0 \leq x \leq 1,
$$

as well as the Dirichlet boundary conditions

$$
f(0, t)=\frac{1}{2} \sin (b \pi t), f(1, t)=\sin (a \pi(t+1)), \quad t>0,
$$

where $a$ and $b$ are constants.

Since conventional difference approximations are consistent to the first derivative [Jain and Sheng 2007; Urban et al. 2004], we may adopt a uniform grid in the temporal direction, while maintaining nonuniform discretization in the $x$-direction. Let $\tau>0$ be the temporal step size used, and $h_{0}, h_{1}, h_{2}, \ldots, h_{n}$ the spatial step sizes, where in general

$$
h_{i} \neq h_{i+1}, \quad i \in\{0,1,2, \ldots, n-1\},
$$

and

$$
\sum_{k=0}^{n} h_{k}=1 .
$$

Thus, a two-dimensional nonuniform grid

$$
D_{h, \tau}=\left\{\left(x_{i}, t_{j}\right): x_{i}=x_{i-1}+h_{i-1}, i=1,2, \ldots, n+1 ; x_{0}=0, x_{n+1}=1\right\}
$$

is a discrete set over the domain $D$. Any $P_{i, j}=\left(x_{i}, t_{j}\right) \in D_{h, \tau}$ is called a grid point of $D_{h, \tau}$. It is an internal grid point if $i \neq 0, n+1$ and $j \neq 0$, a boundary point if $i \in\{0, n+1\}$ and an initial point if $j=0$.

In Figure 1 we show a particular two-dimensional nonuniform grid. In the design of nonuniform grids, we define the smoothness ratios

$$
r_{k}=\frac{h_{k}}{h_{k-1}}, \quad k=1,2, \ldots, n,
$$

and we avoid extreme $r_{k}$ values since they may cause nonphysical oscillations of the numerical solutions inconsistent with the assumption that (1-1) adheres to the principles of geometric Brownian motion [Sheng 2008; Wilmott et al. 1995, §3.5, pp. 41-43].

For concreteness and simplicity, we will fix the parameter values

$$
\alpha=1, \quad \beta=2, \quad \gamma=0
$$

throughout our investigations. 


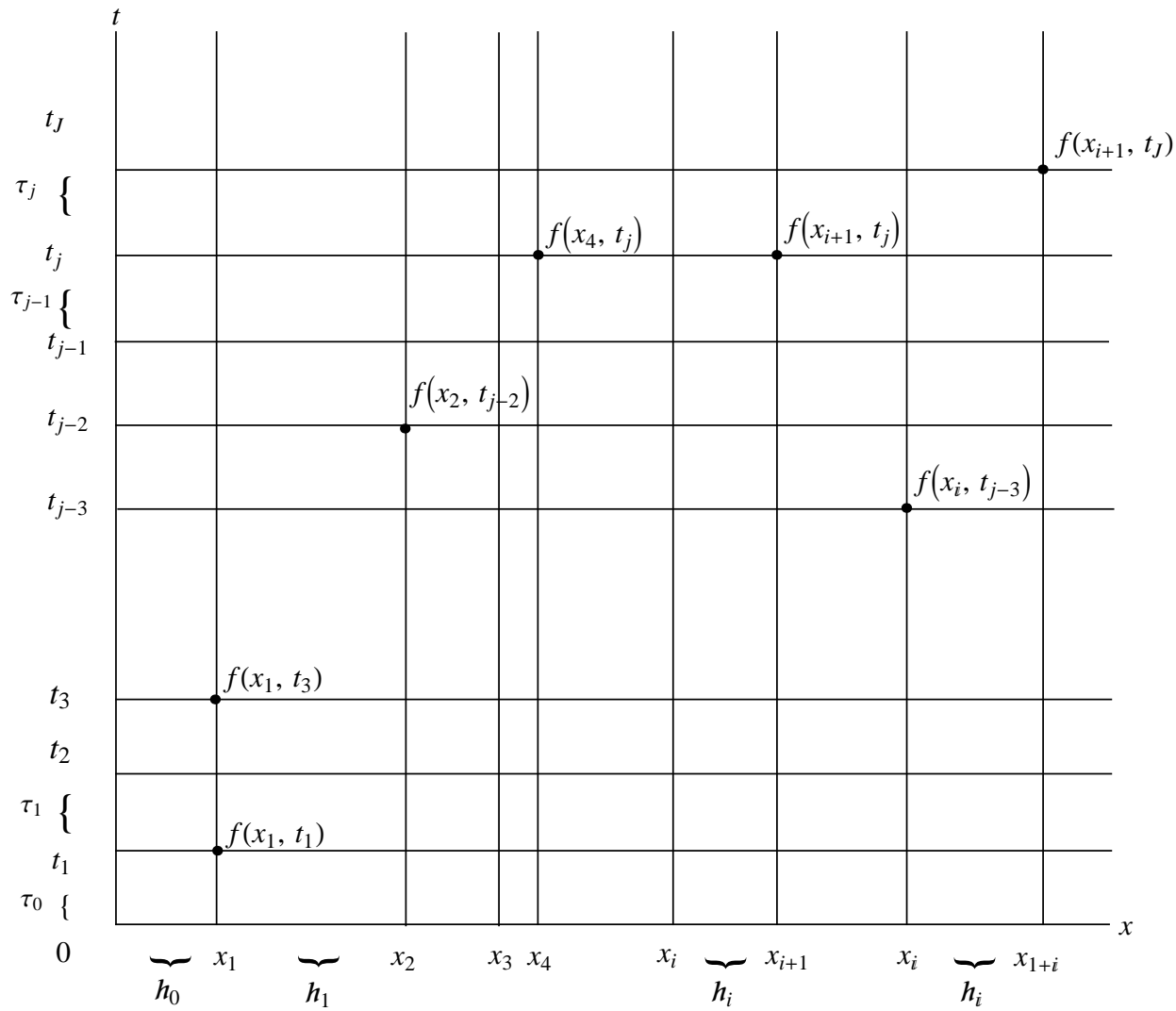

Figure 1. An illustration of the nonuniform grid $D_{h, \tau}$. A temporal step $\tau=0.5$ is used. Particular spatial steps $h_{0}=0.15, h_{1}=0.08$, $h_{2}=0.07, h_{3}=0.06, h_{4}=0.02, h_{5}=0.02, h_{6}=0.01, h_{7}=0.02$, $h_{8}=0.08, h_{9}=0.09, h_{10}=0.08, h_{11}=0.12, h_{12}=0.07, h_{13}=0.1$ are used.

\section{The explicit scheme}

An explicit scheme is an algorithm which can be executed readily, or straightforwardly, without using a system of nonlinear solvers [Atkinson and Han 2004; Sheng 2008; Smith 1985]. In our case, unknowns can be evaluated by first finding the initial values (1-2) and boundary values to the left and right (1-3), and then using those values to arrive at the targeted $f_{i, j}$. We note that the scheme is being taken along a nonuniform grid rather than a uniform grid. In order to find values in the next temporal level, a recursive formula needs to be implemented. The application of computer software is very helpful in computing these $f$ values, where else we would never have been able to see complex numerical results. 
Denote the function value $f\left(x_{i}, t_{j}\right)$ by $f_{i, j}$. From the structure of $D_{h, \tau}$, we know that for any internal grid point $P_{i, j}$ we have

$$
x_{i}=h_{0}+h_{1}+\cdots+h_{i-1}, \quad i \in\{1,2, \ldots, n\} ; \quad t_{j}=j \tau, \quad j>0 .
$$

According to [Atkinson and Han 2004, §4.1, p. 137; Jain and Sheng 2007], at $P_{i, j}$ we have

$$
\begin{aligned}
&\left.\frac{\partial f}{\partial t}\right|_{i, j} \approx \frac{f_{i, j+1}-f_{i, j}}{\tau} \\
& \begin{aligned}
\left.\frac{\partial^{2} f}{\partial x^{2}}\right|_{i, j} & \approx \mathscr{D}_{2, x} f_{i, j}=\frac{D_{x,+} f_{i, j}-D_{x,-} f_{i, j}}{\left(h_{i-1}+h_{i}\right) / 2} \\
& =\frac{2}{h_{i}\left(h_{i-1}+h_{i}\right)} f_{i+1, j}-\frac{1 / h_{i}+1 / h_{i-1}}{\left(h_{i-1}+h_{i}\right) / 2} f_{i, j}+\frac{2}{h_{i-1}\left(h_{i-1}+h_{i}\right)} f_{i-1, j} \\
& =\frac{2}{h_{i}\left(h_{i-1}+h_{i}\right)} f_{i+1, j}-\frac{2}{h_{i-1} h_{i}} f_{i, j}+\frac{2}{h_{i-1}\left(h_{i-1}+h_{i}\right)} f_{i-1, j}
\end{aligned} \\
&\left.\frac{\partial f}{\partial x}\right|_{i, j} \approx \frac{f_{i+1, j}-f_{i-1, j}}{h_{i-1}+h_{i}} .
\end{aligned}
$$

Original concepts of the finite differences (2-1)-(2-3) can be traced back to calculations of variations in $L^{p}$ spaces and beyond [Fonseca and Leoni 2007, §2.1.1].

Recall our choices $\alpha=1, \beta=2, \gamma=0$. If we substitute (2-1)-(2-3) into (1-1) and remove the error terms, we acquire an explicit finite difference equation at $P_{i, j}$ :

$$
\begin{aligned}
B_{h, \tau}\left(f_{i, j}\right)=\frac{f_{i, j+1}-f_{i, j}}{\tau}-\frac{2}{h_{i-1}+} & h_{i}\left(1-\frac{1}{h_{i-1}}\right) f_{i-1, j} \\
& \quad-\frac{2}{h_{i-1} h_{i}} f_{i, j}+\frac{2}{h_{i-1}+h_{i}}\left(1+\frac{1}{h_{i}}\right) f_{i+1, j}=0,
\end{aligned}
$$

where $B_{h, \tau}$ can be viewed as a discretized Black-Scholes operator. The above can be reformulated to the recursive formula

$$
\begin{aligned}
f_{i, j+1} & =f_{i, j}+\sigma_{i}\left[\left(1-\frac{1}{h_{i-1}}\right) f_{i-1, j}+\frac{h_{i-1}+h_{i}}{h_{i-1} h_{i}} f_{i, j}-\left(1+\frac{1}{h_{i}}\right) f_{i+1, j}\right], \\
\sigma_{i} & =\frac{2 \tau}{h_{i-1}+h_{i}}
\end{aligned}
$$

or

$$
\begin{aligned}
f_{i, j+1} & =\sigma_{i}\left(1-\frac{1}{h_{i-1}}\right) f_{i-1, j}+\left(1+\frac{2 \tau}{h_{i-1} h_{i}}\right) f_{i, j}-\sigma_{i}\left(1+\frac{1}{h_{i}}\right) f_{i+1, j}, \\
\sigma_{i} & =\frac{2 \tau}{h_{i-1}+h_{i}},
\end{aligned}
$$

which runs for the temporal level index $j$ from 0 to $J$, as far as $J \tau \leq T$. The numerical solution can thus be derived from the recursive relation (2-4) together 
with the initial and boundary conditions (1-2), (1-3). This explicit finite difference scheme (2-4), (1-2), (1-3) is originally presented in [Sheng 2008].

To analyze (2-4) we need the following definitions:

Definition 2.1 (Order of accuracy). Consider a discretized Black-Scholes operator $B_{h, \tau}$, where $0<h, \tau<1$. Assume that the function $f(x, t)$ is sufficiently smooth (no cusps or discontinuities of partial derivatives up to a desired order) over $D$. If

$$
\max _{\left(x_{i}, t_{j}\right) \in D_{h, \tau}}\left|B(f)\left(x_{i}, t_{j}\right)-B_{h, \tau}\left(f_{i, j}\right)\right|=O\left(h^{p}+\tau^{q}\right),
$$

where $h=\max \left\{h_{1}, h_{2}, \ldots, h_{N}\right\}$, we say that the difference scheme defined by $B_{h, \tau}$ is an $\operatorname{order}-(p, q)$ scheme for solving the Black-Scholes equation (1-1). We also say that $B_{h, \tau}$ is an order-r scheme, where $r=\min \{p, q\}$.

A difference method is practically meaningful when both $p$ and $q$ are positive. Definition 2.2. A difference method $B_{h, \tau}$ is consistent if it has order $r>0$.

There is always an error associated with a finite difference approximation. If we use Taylor's Theorem to expand certain known finite difference approximations along difference schemes, such as (2-4), we can empirically prove that these approximations may or may not be useful when applied to the Black-Scholes equation. For this purpose, we need the following notion:

Definition 2.3. The truncation error function of the difference scheme is defined as

$$
\operatorname{err}(f)_{i, j}=B(f)\left(x_{i}, t_{j}\right)-B_{h, \tau}\left(f_{i, j}\right), \quad\left(x_{i}, t_{j}\right) \in D_{h, \tau} .
$$

Theorem 2.4. For the explicit finite difference scheme (2-4) we have the truncation error estimate

$$
\operatorname{err}_{E}(f)_{i, j}=O(h+\tau),
$$

where $h=\max \left\{h_{1}, h_{2}, \ldots, h_{N}\right\}$. Therefore the explicit scheme (2-4) is of first order.

If the local spatial grid is uniform, that is, $h_{i}=h_{i-1}=h>0$, the scheme (2-4) is locally of second order in space:

$$
\operatorname{err}_{E}(f)_{i, j}=O\left(h^{2}+\tau\right) .
$$

Proof. We use the so-called forward, central and modified central difference operators, defined respectively by

$$
\Delta_{t} f=\frac{f\left(t_{i, j+1}\right)-f\left(t_{i, j}\right)}{\tau_{i, j+1}-\tau_{i, j}}, \quad \delta_{x} f=\frac{f\left(x_{i+1, j}\right)-f\left(x_{i-1, j}\right)}{h_{i+1, j}-h_{i-1, j}}, \mathscr{D}_{2, x} f=\frac{\Delta_{x} f-\nabla_{x} f}{\left(h_{i, j-1}+h_{i, j}\right) / 2},
$$

for $i \in X, j \in T$. In the last expression $\Delta_{x}$ is defined like $\Delta_{t}$, and the backward spatial difference operator $\nabla_{x}$ is similar, but with all spatial indices decreased by 1 . In this notation we obtain from (2-4) 


$$
\operatorname{err}_{E}(f)_{i, j}=\left(f_{t}-\Delta_{t} f\right)_{i, j}+\alpha\left(f_{x x}-\mathscr{D}_{2, x} f\right)_{i, j}+\beta\left(f_{x}-\delta_{x} f\right)_{i, j} .
$$

We have (see [Jain and Sheng 2007] for details)

$$
\begin{gathered}
\left(f_{t}-\Delta_{t} f\right)_{i, j}=-\frac{1}{2} \tau f_{t t}\left(x_{i}, t_{j}\right)-\frac{1}{6} \tau^{2} f_{t t t}\left(x_{i}, t_{j}\right)+\cdots, \\
\left(f_{x x}-\mathscr{D}_{2, x} f\right)_{i, j}=-\frac{1}{3}\left(h_{i}-h_{i-1}\right) f_{x x x}\left(x_{i}, t_{j}\right) \\
\quad-\frac{1}{12}\left(h_{i}^{2}-h_{i} h_{i-1}+h_{i-1}^{2}\right) f_{x^{4}}\left(x_{i}, t_{j}\right)-\cdots, \\
\left(f_{x}-\delta_{x} f\right)_{i, j}=-\frac{1}{2\left(h_{i}+h_{i-1}\right)}\left(h_{i}^{2} f_{x x}\left(x_{i}, t_{j}\right)-h_{i-1}^{2} f_{x x}\left(x_{i}, t_{j}\right)+\cdots\right) \\
=\frac{1}{2}\left(h_{i}-h_{i-1}\right) f_{x x}\left(x_{i}, t_{j}\right)+\frac{1}{6}\left(h_{i}^{2}-h_{i} h_{i-1}+h_{i-1}^{2}\right) f_{x x x}\left(x_{i}, t_{j}\right)+\cdots .
\end{gathered}
$$

Thus the lowest-order terms are linear in $\max \left\{h_{i}, h_{i+1}\right\}$ and $\tau_{j}$; but when $h_{i}=$ $h_{i+1}=h$, the linear contributions in $h$ drop out.

\section{The implicit scheme}

Instead of the forward finite difference (2-1), we may consider the backward difference formula

$$
\left.\frac{\partial f}{\partial t}\right|_{i, j}=\frac{f_{i, j}-f_{i, j-1}}{\tau}+O(\tau) .
$$

Substitution of (3-1), (2-2), and (2-3) into (1-1) yields

$$
\begin{aligned}
\frac{f_{i, j}-f_{i, j-1}}{\tau}=\frac{2}{h_{i-1}+h_{i}}\left(1-\frac{1}{h_{i-1}}\right) & f_{i-1, j} \\
& +\frac{2}{h_{i-1} h_{i}} f_{i, j}-\frac{2}{h_{i-1}+h_{i}}\left(1+\frac{1}{h_{i}}\right) f_{i+1, j},
\end{aligned}
$$

which is significantly different from the explicit scheme (2-4).

For later convenience we replace the index $j$ by $j+1$ and $j-1$ by $j$. Our difference equation then becomes

$$
\begin{aligned}
\frac{f_{i, j+1}-f_{i, j}}{\tau}=\frac{2}{h_{i-1}+h_{i}}\left(1-\frac{1}{h_{i-1}}\right) & f_{i-1, j+1} \\
& \quad+\frac{2}{h_{i-1} h_{i}} f_{i, j+1}-\frac{2}{h_{i-1}+h_{i}}\left(1+\frac{1}{h_{i}}\right) f_{i+1, j+1},
\end{aligned}
$$

which can further be written as

$$
-\sigma_{i}\left(1-\frac{1}{h_{i-1}}\right) f_{i-1, j+1}-\left(\frac{2 \tau}{h_{i-1} h_{i}}-1\right) f_{i, j+1}+\sigma_{i}\left(1+\frac{1}{h_{i}}\right) f_{i+1, j+1}=f_{i, j},
$$

where $\sigma_{i}$ is the same as defined before. The implicit finite difference algorithm (3-2), together with conditions (1-2), (1-3), is studied in [Sheng 2008].

Equation (3-2) cannot be solved independently without collaboration between the rest of the equations at the temporal level $j+1$. We note that there are $n$ internal 
grid points. Thus we have $n$ difference equations at each of the temporal levels, and we get a linear system of size $n$, which can be expressed in the matrix form as

$$
M f_{j+1}=g_{j+1}, \quad j \in\{1,2, \ldots, J\},
$$

where $M$ is a tridiagonal matrix with nontrivial elements

$$
\begin{array}{ll}
m_{i, i+1}=-\sigma_{i}\left(1+1 / h_{i}\right), & i=1,2, \ldots, n-1, \\
m_{i, i}=2 \tau /\left(h_{i-1} h_{i}\right)-1, & i=1,2, \ldots, n, \\
m_{i, i-1}=\sigma_{i}\left(1-1 / h_{i-1}\right), & i=2,3, \ldots, n .
\end{array}
$$

For the vectors we have

$$
\begin{aligned}
& \left(f_{j+1}\right)_{i}=f_{i, j+1}, \quad i=1,2, \ldots, n, \\
& \left(g_{j+1}\right)_{1}=-f_{1, j}-\sigma_{1}\left(1-1 / h_{0}\right) f_{0, j+1}, \\
& \left(g_{j+1}\right)_{i}=-f_{i, j}, \quad i=2,3, \ldots, n-1, \\
& \left(g_{j+1}\right)_{n}=-f_{n, j}+\sigma_{n}\left(1+1 / h_{n}\right) f_{n+1, j+1},
\end{aligned}
$$

where the values of $f_{0, j+1}$ and $f_{n+1, j+1}$ are given by the condition (1-3).

The tridiagonal system of linear equations (3-3) can be solved conveniently by using a special subroutine in C and Matlab; see [Jain and Sheng 2007; Pratap 1999; Sheng 2008].

Theorem 3.1. For the implicit finite difference scheme (3-2) or (3-3) we have the truncation error estimate

$$
\operatorname{err}_{I}(f)_{i, j}=O(h+\tau),
$$

where $h=\max \left\{h_{1}, h_{2}, \ldots, h_{N}\right\}$. Therefore the implicit scheme is of first order.

If the local spatial grid region is uniform, that is, $h_{i}=h_{i-1}=h>0$, the scheme is locally of second order in space:

$$
\operatorname{err}_{I}(f)_{i, j}=O\left(h^{2}+\tau\right) .
$$

Proof. We have

$$
\operatorname{err}_{I}(f)_{i, j}=\left(f_{t}-\nabla_{t} f\right)_{i, j}+\alpha\left(f_{x x}-\mathscr{D}_{2, x} f\right)_{i, j}+\beta\left(f_{x}-\delta_{x} f\right)_{i, j} .
$$

The $\alpha$ and $\beta$ terms are as in the proof of Theorem 2.4, while for the remaining term we have (from [Jain and Sheng 2007], for instance).

$$
\left(f_{t}-\nabla_{t} f\right)_{i, j}=\frac{1}{2} \tau f_{t t}\left(x_{i}, t_{j}\right)-\frac{1}{6} \tau^{2} f_{t t t}\left(x_{i}, t_{j}\right)+\cdots .
$$

Substitution into (3-4) and consideration of the special case $h_{i}=h_{i-1}$ yields the result. 


\section{The leapfrog scheme}

In this much more sophisticated approach, we first replace (2-1) by the central difference formula

$$
\left.\frac{\partial f}{\partial t}\right|_{i, j}=\frac{f_{i, j+1}-f_{i, j-1}}{2 \tau}+O\left(\tau^{2}\right) .
$$

We immediately notice the increase in the order of approximation. Now, instead of (2-2), (2-3) for the spatial derivatives, we consider the average formulas

$$
\begin{gathered}
\left.\frac{\partial^{2} f}{\partial x^{2}}\right|_{i, j}=\frac{1}{2}\left(\frac{D_{x,+} f_{i, j-1}-D_{x,-} f_{i, j-1}}{\left(h_{i-1}+h_{i}\right) / 2}+\frac{D_{x,+} f_{i, j+1}-D_{x,-} f_{i, j+1}}{\left(h_{i-1}+h_{i}\right) / 2}\right)+O(h) \\
=\frac{1}{h_{i}\left(h_{i-1}+h_{i}\right)}\left(f_{i+1, j-1}+f_{i+1, j+1}\right)-\frac{1}{h_{i-1} h_{i}}\left(f_{i, j-1}+f_{i, j+1}\right) \\
+\frac{1}{h_{i-1}\left(h_{i-1}+h_{i}\right)}\left(f_{i-1, j-1}+f_{i-1, j+1}\right)+O(h), \\
\left.\frac{\partial f}{\partial x}\right|_{i, j}=\frac{1}{2}\left(\frac{f_{i+1, j-1}-f_{i-1, j-1}}{h_{i-1}+h_{i}}+\frac{f_{i+1, j+1}-f_{i-1, j+1}}{h_{i-1}+h_{i}}\right)+O(h),
\end{gathered}
$$

where $h=\max _{k}\left\{h_{k}\right\}$. The order of approximation of the spatial derivatives is still 1 due to the nonuniform grid.

Substitution of (4-1)-(4-3) into (1-1) yields

$$
\begin{aligned}
\frac{f_{i, j+1}-f_{i, j-1}}{2 \tau}=\frac{1}{h_{i-1}+h_{i}}\left(1-\frac{1}{h_{i}}\right) & \left(f_{i-1, j-1}+f_{i-1, j+1}\right)+\frac{1}{h_{i-1} h_{i}}\left(f_{i, j-1}+f_{i, j+1}\right) \\
& -\frac{1}{h_{i-1}+h_{i}}\left(1+\frac{1}{h_{i}}\right)\left(f_{i+1, j-1}+f_{i+1, j+1}\right) .
\end{aligned}
$$

Let

$$
\sigma_{i}=\frac{2 \tau}{h_{i-1}+h_{i}} .
$$

Then the difference equation can be rearranged as

$$
\begin{aligned}
f_{i, j+1}=f_{i, j-1}+ & \sigma_{i}\left(1-\frac{1}{h_{i}}\right)\left(f_{i-1, j-1}+f_{i-1, j+1}\right) \\
& +\frac{2 \tau}{h_{i-1} h_{i}}\left(f_{i, j-1}+f_{i, j+1}\right)-\sigma_{i}\left(1+\frac{1}{h_{i}}\right)\left(f_{i+1, j-1}+f_{i+1, j+1}\right),
\end{aligned}
$$

which leads to

$$
\begin{aligned}
\sigma_{i}\left(1-\frac{1}{h_{i}}\right) f_{i-1, j+1}+\left(\frac{2 \tau}{h_{i-1} h_{i}}-1\right) f_{i, j+1}-\sigma_{i}\left(1+\frac{1}{h_{i}}\right) f_{i+1, j+1} \\
\quad=-\sigma_{i}\left(1-\frac{1}{h_{i}}\right) f_{i-1, j-1}-\left(\frac{2 \tau}{h_{i-1} h_{i}}+1\right) f_{i, j-1}+\sigma_{i}\left(1+\frac{1}{h_{i}}\right) f_{i+1, j-1} .
\end{aligned}
$$


Like(3-2), the system of linear equations (4-4) can be put into matrix form:

$$
P f_{j+1}=Q f_{j-1}+s_{j+1},
$$

where $P, Q$ are $n \times n$ tridiagonal matrices and $s_{j+1}$ can be determined via the boundary condition (1-3). Therefore (4-5) can be readily solved by computers [Atkinson and Han 2004; Sheng 2008; Smith 1985].

The leapfrog scheme (4-4), or (4-5), is implicit since it must be solved as a linear system.

A peculiarity of the leapfrog method is that if we start from the initial value at $t=0$, we can only obtain the numerical solution of (1-1)-(1-3) on even temporal levels. To compute numerical solutions on odd temporal levels, we need solution values on the first temporal level, which can be generated by using one step via either the explicit method or implicit method.

Theorem 4.1. For the leapfrog implicit finite difference scheme (4-4) or (4-5) we have the truncation error estimate:

$$
\operatorname{err}_{L}(f)_{i, j}=O\left(h+\tau^{2}\right)
$$

where

$$
h=\max \left\{h_{1}, h_{2}, \ldots, h_{N}\right\} .
$$

Therefore the leapfrog scheme is of first order in space and second order in time.

If the local spatial grid region is uniform, that is, $h_{i}=h_{i-1}=h>0$, the leapfrog scheme becomes a second order method locally:

$$
\operatorname{err}_{L}(f)_{i, j}=O\left(h^{2}+\tau^{2}\right)
$$

Proof. For the leapfrog scheme (4-4) or (4-5),

$$
\begin{array}{r}
\operatorname{err}_{L}(f)_{i, j}=\left(f_{t}-\delta_{t} f\right)_{i, j}+\alpha\left(\left(f_{x x}\right)_{i, j}-\frac{1}{2}\left(\mathscr{D}_{2, x} f_{i, j-1}+\mathscr{D}_{2, x} f_{i, j+1}\right)\right) \\
+\beta\left(\left(f_{x}\right)_{i, j}-\frac{1}{2}\left(\delta_{x} f_{i, j-1}+\delta_{x} f_{i, j+1}\right)\right) .
\end{array}
$$

Again, according to [Jain and Sheng 2007; Sheng 2008], we have

$$
\left(f_{t}-\delta_{t} f\right)_{i, j}=-\frac{1}{6} \tau^{2} f_{t t t}\left(x_{i}, t_{j}\right)-\frac{1}{120} \tau^{4} f_{t^{5}}\left(x_{i}, t_{j}\right)-\cdots .
$$

Note that, since the grid distribution in space is irregular, (4-7) cannot be extended for estimating the difference $\left(f_{x}\right)_{i, j}-\frac{1}{2}\left(\delta_{x} f_{i, j-1}+\delta_{x} f_{i, j+1}\right)$. Instead, employing the expansion

$\delta_{x} f_{i, j-1}=\left(f_{x}\right)_{i, j-1}+\frac{1}{2}\left(h_{i}-h_{i-1}\right)\left(f_{x x}\right)_{i, j-1}+\frac{1}{6}\left(h_{i}^{2}-h_{i} h_{i-1}+h_{i-1}^{2}\right)\left(f_{x x x}\right)_{i, j-1}+\cdots$ 
and performing simplifications, we obtain

$$
\begin{aligned}
& \left(f_{x}\right)_{i, j}-\frac{1}{2}\left(\delta_{x} f_{i, j-1}+\delta_{x} f_{i, j+1}\right) \\
& =-\frac{1}{4}\left(h_{i}-h_{i-1}\right)\left(\left(f_{x x}\right)_{i, j-1}+\left(f_{x x}\right)_{i, j+1}\right) \\
& \quad-\frac{1}{12}\left(h_{i}^{2}-h_{i} h_{i-1}+h_{i-1}^{2}\right)\left(\left(f_{x x x}\right)_{i, j-1}+\left(f_{x x x}\right)_{i, j+1}\right)+O\left(h^{3}\right) .
\end{aligned}
$$

For the $\alpha$ term in (4-7) we will assume for simplicity that the grids $\left\{x_{i}\right\}$ remain the same on different temporal levels. Using the expansion

$$
\mathscr{D}_{2, x} f_{i, j-1}=\frac{1}{3}\left(h_{i}-h_{i-1}\right) 3\left(f_{x x x}\right)_{i, j-1}+\frac{1}{12}\left(h_{i}^{2}-h_{i} h_{i-1}+h_{i-1}^{2}\right)\left(f_{x^{4}}\right)_{i, j-1}+\cdots
$$

and simplifying, we obtain

$$
\begin{aligned}
& \left(f_{x x}\right)_{i, j}-\frac{1}{2}\left(\mathscr{D}_{2, x} f_{i, j-1}+\mathscr{D}_{2, x} f_{i, j+1}\right) \\
& =-\frac{1}{6}\left(h_{i}-h_{i-1}\right)\left(\left(f_{x x x}\right)_{i, j-1}+3\left(f_{x x x}\right)_{i, j}+\left(f_{x x x}\right)_{i, j+1}\right) \\
& -\frac{1}{24}\left(h_{i}^{2}+h_{i-1}^{2}\right)\left(\left(f_{x^{4}}\right)_{i, j-1}+6\left(f_{x^{4}}\right)_{i, j}+\left(f_{x x x}\right)_{i, j+1}\right) \\
& +\frac{1}{24} h_{i} h_{i-1}\left(\left(f_{x^{4}}\right)_{i, j-1}+\left(f_{x^{4}}\right)_{i, j+1}\right)+O\left(h^{3}\right) .
\end{aligned}
$$

Substituting (4-7)-(4-9) into (4-6) we get for $\operatorname{err}_{L}(f)_{i, j}$ the expression

$$
\begin{aligned}
-\frac{1}{6} \tau^{2} f_{t t t}\left(x_{i}, t_{j}\right)+\alpha\left[\frac{1}{6}\left(h_{i}-h_{i-1}\right)\left(\left(f_{x x x}\right)_{i, j-1}+3\left(f_{x x x}\right)_{i, j}+\left(f_{x x x}\right)_{i, j+1}\right)\right. \\
+\frac{1}{24}\left(h_{i}^{2}+h_{i-1}^{2}\right)\left(\left(f_{x^{4}}\right)_{i, j-1}+6\left(f_{x^{4}}\right)_{i, j}+\left(f_{x^{4}}\right)_{i, j+1}\right) \\
\left.+\frac{1}{24} h_{i} h_{i-1}\left(\left(f_{x^{4}}\right)_{i, j-1}+\left(f_{x^{4}}\right)_{i, j+1}\right)\right] \\
+\beta\left[\frac{1}{4}\left(h_{i}-h_{i-1}\right)\left(\left(f_{x x}\right)_{i, j-1}+\left(f_{x x}\right)_{i, j+1}\right)\right. \\
\left.+\frac{1}{12}\left(h_{i}^{2}-h_{i} h_{i-1}+h_{i-1}^{2}\right)\left(\left(f_{x x x}\right)_{i, j-1}+\left(f_{x x x}\right)_{i, j+1}\right)\right] \\
+O\left(h^{3}+\tau^{4}\right),
\end{aligned}
$$

which is generally of first order in space and second order in time, but becomes of second order in both time and space when $h_{i}=h_{i-1}$.

Since a leapfrog scheme spans three temporal levels, the computation using (4-4) or (4-5) can be started initially. One strategy is to use an implicit or an explicit scheme for calculating the numerical solution at the first temporal level, that is, when $j=1$. Then, by using the numerical solutions at temporal levels 0 and 1 , a leapfrog scheme can generate solutions at higher temporal levels.

However, this treatment may reduce the overall order of accuracy, given that an explicit or implicit method is used to generate the solution on the first temporal level. The computer club members had several fruitful discussions on the issue. At the end, we realized that such a computation is not necessary. Let $\tau$ be halved. Why not collect numerical solutions on the even number of temporal levels only? 
This will guarantee our overall accuracy. We may introduce imaginary middle temporal grid points [Atkinson and Han 2004; Sheng 2008; Smith 1985] in the analysis, which may help to retain the overall second order accuracy in time $t$. We will report details elsewhere.

\section{Simulation results}

In our numerical experiments, we consider the following simplified Black-Scholes initial-boundary value problem [Brandimarte 2006]:

$$
\begin{aligned}
\frac{\partial f}{\partial t}(x, t) & =-\frac{\partial^{2} f}{\partial x^{2}}(x, t)-2 \frac{\partial f}{\partial x}(x, t), & & 0 \leq x \leq 1, t \geq 0, \\
f(x, 0) & =\sin (2.2 \pi x), & & 0 \leq x \leq 1, \\
f(0, t) & =0.5 \sin (7 \pi t), & & t>0 . \\
f(1, t) & =\sin (2.2 \pi t), & & \text { r }
\end{aligned}
$$

For the sake of simplicity, we will only provide numerical results from the explicit scheme (2-4). Results from the other two schemes are similar.

Our nonuniform grid region is designed as follows: Let $N=100$ and use $\mathrm{C}$ and Matlab programs to generate $N$ random numbers, $x_{1}<x_{2}<x_{3}<\cdots<x_{N}$, on the interval $(0,1)$. Denote $x_{0}=0, x_{N+1}=1$. We have the set of nonuniform spatial step sizes:

$$
h_{i}=x_{i}-x_{i-1}, \quad i=1,2, \ldots, N+1 .
$$

Now, set $\tau=1 / 100$. Thus a nonuniform two-dimensional grid region is completed. In Figure 2, we show the spatial step sizes across the interval $[0,1]$. We also show the ratio distribution of the neighboring spatial step sizes, $h_{i+1} / h_{i}, i=1,2, \ldots, N$,
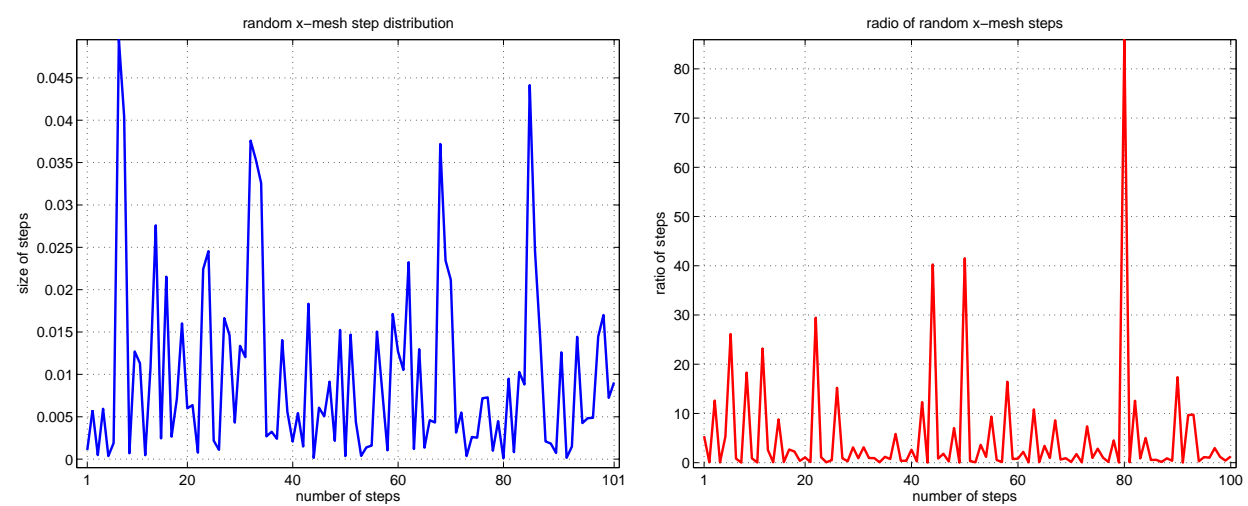

Figure 2. Left: distribution of the random spatial step sizes. Right: ratio function value distribution across the interval $[0,1]$. 
since the values are important in adaptive computations [Jain and Sheng 2007]. Note the large peak value of the ratio function near $i=80$, exemplifying the socalled nonsmoothness phenomenon of random spatial grids [Jain and Sheng 2007; Sheng 2008]. Because the step sizes are random, each numerical experiment with the same Black-Scholes problem (5-1)-(5-3) yields a different Figure 2.

We choose the smooth test function $f(x, t)=\sin (\pi x) e^{-0.22 t}$, as in [Atkinson and Han 2004; Jain and Sheng 2007]. Its partial derivatives are readily calculated. Figure 3 shows illustrative plots of the finite difference approximations for $f$ and its derivatives. The $\mathscr{D}_{2, x}$ formula is used in approximating the second derivative since repeated use of conventional first order differences does not yield a consistent approximation of the second derivative [Fonseca and Leoni 2007; Jain and Sheng 2007]. For ease of comparison, all function surfaces are plotted from the same viewpoint.
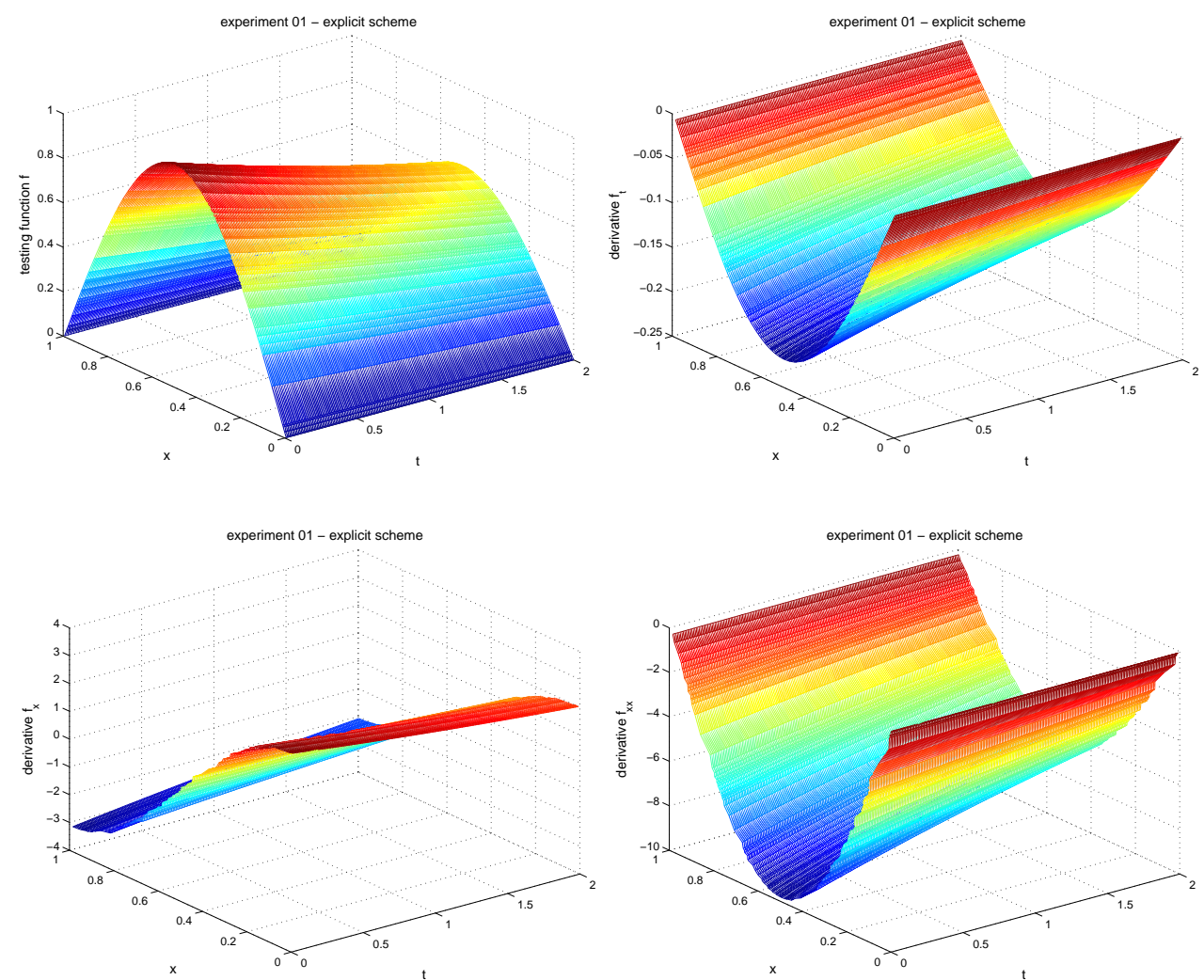

Figure 3. Clockwise from top left: graphs of $f(x, t), f_{t}(x, t)$, $f_{x x}(x, t)$ (similar to $\left.f_{t}(x, t)\right)$ and $f_{x}(x, t)$. Second derivatives use the $\mathscr{D}_{2, x}$ formula. 

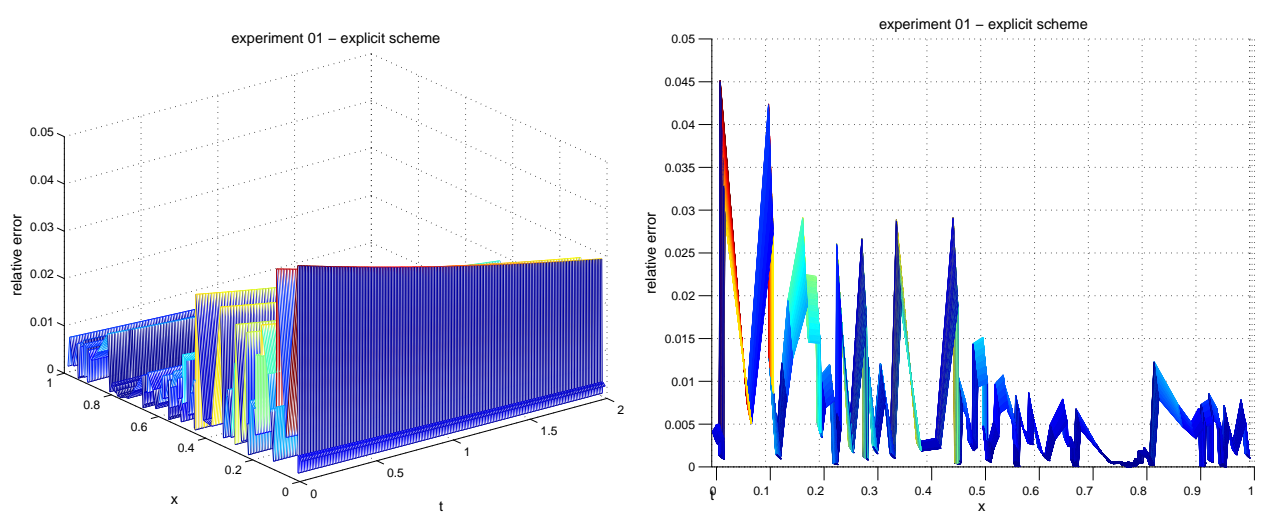

Figure 4. Left: three-dimensional distribution of the relative numerical error. Right: projection of the graph onto the $X Z$ plane.

Consider the explicit scheme (2-4). To check the numerical truncation error of the algorithm, we submit the function $f(x, t)$ into $(2-6)$ and evaluate the outcome on all internal nonuniform grid points. The relative error is adopted for a more reasonable evaluation of the errors [Atkinson and Han 2004].

Figure 4 gives the error distribution over the nonuniform grids of the domain $0 \leq x \leq 1,0 \leq t \leq 2$. In the first figure, we show a three-dimensional relative error distribution. The second figure represents the numerical error projected onto the $X Z$ plane so that we can view more clearly the oscillatory features of the error pattern (probably due to the random spatial steps used). The overall numerical error is oscillatory but small. The maximal relative error appears to be approximately 0.045 , that is, $4.5 \%$, which is satisfactory. It is interesting that the error pattern does not seem to be similar to the mesh pattern given in Figure 2, though they must be related. A more in-depth numerical analysis may be required to reveal their internal connections.

The numerical experiments for estimating the order of convergence is more complex and tricky too, since the feature of two-dimensional problems (5-1)-(5-3) and the nonuniform grids used. Our experiments are based on the following stages:

(1) For a given testing function $f(x, t)$, let the numerical truncation error function be $\left(\operatorname{err}_{0}\right)_{i, j}$. Since $h_{i}=O(\tau)$, we may assume that

$$
\left|\left(\operatorname{err}_{0}\right)_{i, j}\right| \approx M \tau^{p},
$$

where $M$ is a positive constant, for all valid indexes $i, j$.

(2) Halve both the spatial and temporal step sizes. This is easy to achieve in time but relatively tricky in space. For the sake of simplicity, we may halve each of the $h_{i}$ generated, although this yields pairs of identical step sizes. 

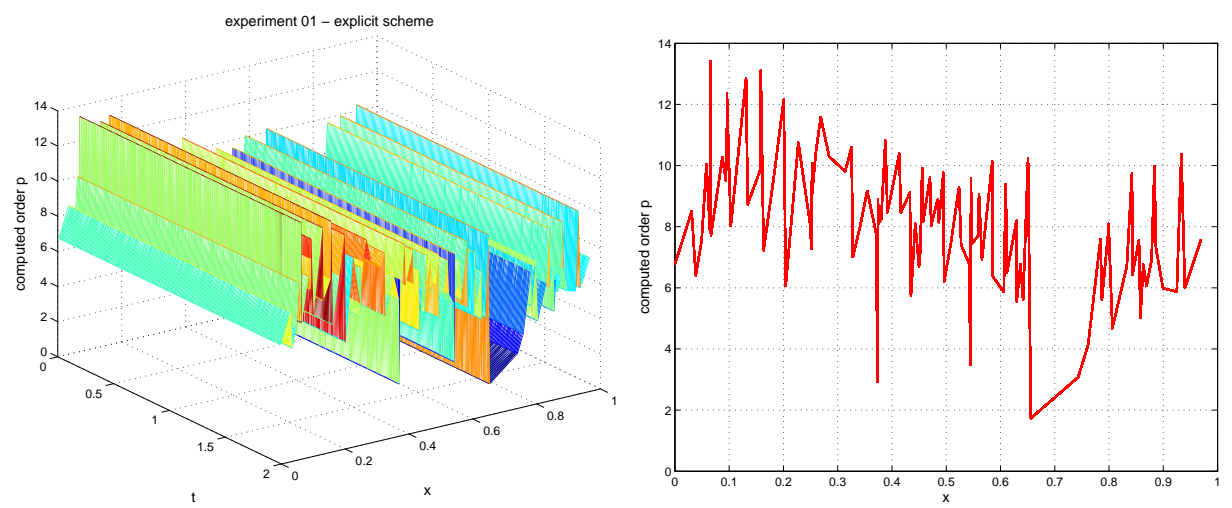

Figure 5. Left: three-dimensional distribution of the order function $p$. Right: contour map of the function $p$ over the region $0 \leq x \leq 1,0 \leq t \leq 2$.

(3) Repeat the computation on the refined grid region. Suppose that the new numerical truncation error function is $\left(\operatorname{err}_{1}\right)_{i, j}$, where the indexes are taken only on grid points where $\left(\operatorname{err}_{0}\right)_{i, j}$ is defined. Thus, we have

$$
\left|\left(\operatorname{err}_{1}\right)_{i, j}\right| \approx M(\tau / 2)^{p}
$$

for all such indexes $i, j$.

(4) From (5-4), (5-5) we deduce that $\left|\frac{\left(\operatorname{err}_{0}\right)_{i, j}}{\left(\operatorname{err}_{1}\right)_{i, j}}\right| \approx 2^{p}$, which offers an estimate

$$
p \approx \frac{1}{\ln 2} \ln \left|\frac{\left(\mathrm{err}_{0}\right)_{i, j}}{\left(\operatorname{err}_{1}\right)_{i, j}}\right| \text {. }
$$

Evidently, such a $p$ is also a function of $i, j$. Therefore an average value of such $p$ values would provide a more reasonable estimate of the order for the underlying numerical method.

The process may be repeated by halving the step sizes again. However, note that (5-6) provides only an estimate which can be used as a reference.

Figure 5 demonstrates a solution surface of the $p$ values obtained via (5-6). It is interesting to notice that

$$
\max _{i, j} p=13.4235, \quad \min _{i, j} p=1.7357, \quad \text { average }(p)=8.1013 .
$$

The numerical results seem to be much higher than the linear truncation error predicted by Theorem 2.4 in the situation. Most of our randomly chosen $x$-grids have demonstrated a similar conclusion. However, since the testing function is artificially chosen and the grid points in the $x$-direction are randomly chosen, we cannot conclude that the actual truncation order is much better than predicted. The 
experiment results only indicate a strong possibility that our numerical scheme may behave better than anticipated.

\section{Conclusion}

Financial confidence is of the utmost importance when making investments, and in this paper we analyze the consistency of explicit, implicit and leapfrog finite difference schemes for solving Black-Scholes partial differential equation. We show the potential these numerical methods have for making accurate predictions of the option values when nonuniform discretizations are necessary. By using the $\mathscr{D}_{2, x}$ difference formula, we prove that all difference methods developed are consistent. While the explicit and implicit schemes provide a truncation error of the order $O(\tau+h)$, the leapfrog scheme offers a higher order $O\left(\tau^{2}+h\right)$. More precise error estimates for the three numerical methods are delivered in the corollaries for closer comparisons. Numerical experiments based on the explicit finite difference scheme have demonstrated a satisfactory result, indicating their good potential use in real-world implementations.

The orders of approximation can be further improved, but, the use of more sophisticated finite difference formulas may lead to complicated numerical schemes which are either difficult to use or difficult to analyze. The benefit of order improvement may be limited in actual financial computations, though its theory in numerical investigations is always meaningful. The exploration of better, higherorder numerical schemes for solving the Black-Scholes equation is one of the goals in our forthcoming study.

There are many interesting problems to be explored for the finite difference schemes implemented. A particularly relevant issue is numerical stability in the von Neumann sense [Atkinson and Han 2004; Sheng 2008; Smith 1985]. This concern focuses on the question: once a tiny error is introduced during computations, will it affect significantly further option values of $f$ ? If such a consequence is unavoidable, then is there a strategy to reduce the damage? We prefer to leave the answers to our forthcoming investigations. We also encourage the reader to explore any possible solutions.

\section{Acknowledgments}

The authors are grateful to Dr. Johnny Henderson and Dr. Lance Littlejohn, professors of mathematics at Baylor University, and Mr. Andrew D. Sheng, undergraduate student of computer science at Carnegie Mellon University, for reading our manuscripts, constructing and experimenting many programs via $\mathrm{C}, \mathrm{C}++$ and Matlab languages, and for sharing important research notes and thoughts. Our 
special thanks go to Baylor University for its Undergraduate Research and Scholarly Achievement (URSA) grant, which enabled this collaborative research. We also thank our project advisor, Dr. Qin Sheng, professor of mathematics at Baylor University, for suggesting this line of undergraduate research and for the encouragement and numerous discussions throughout the project. Last, but not least, we appreciate our reviewers for their comments and suggestions that helped to improve the contents of this paper.

\section{References}

[Atkinson and Han 2004] K. Atkinson and W. Han, Elementary numerical analysis, 3rd ed., Wiley, Somerset, NJ, 2004. Zbl 0782.65002

[Brandimarte 2006] P. Brandimarte, Numerical methods in finance and economics: A MATLABbased introduction, 2nd ed., Wiley, Hoboken, NJ, 2006. MR 2007d:91001 Zbl 1129.91002

[Fonseca and Leoni 2007] I. Fonseca and G. Leoni, Modern methods in the calculus of variations: $L^{p}$ spaces, Springer, New York, 2007. MR 2008m:49001 Zbl 1153.49001

[Jain and Sheng 2007] B. Jain and A. D. Sheng, "An exploration of the approximation of derivative functions via finite differences", Rose-Hulman Undergraduate Math Journal 8 (2007), 172-188.

[Pratap 1999] R. Pratap, Getting started with MatLab 5, Oxford University Press, Oxford and New York, 1999.

[Sheng 2008] A. D. Sheng, "Optimized finite difference approximations on non-uniform grids with applications: A midterm project report to the URSA program”, preprint, Baylor University, 2008, Available at http://www.baylor.edu/research/ursa/index.php?id $=50690$.

[Smith 1985] G. D. Smith, Numerical solution of partial differential equations: Finite difference methods, 3rd ed., Oxford App. Math. and Computing Science Series 13, Oxford Univ. Press, New York, 1985. MR 87c:65002 Zbl 0576.65089

[Urban et al. 2004] P. Urban, J. Owen, D. Martin, R. Haese, S. Haese, and M. Bruce, Mathematics for the international student : Mathematics HL (core), Haese \& Harris Publications, Adelaide Airport, Australia, 2004.

[Wilmott et al. 1995] P. Wilmott, S. Howison, and J. Dewynne, The mathematics of financial derivatives: A student introduction, Cambridge University Press, Cambridge, 1995. MR 96h:90028 Zbl 0842.90008

Received: 2009-06-04 Revised: 2009-07-23 Accepted: 2009-07-27

mylesdanielbaker@gmail.com Department of Mathematics, Baylor University, Waco, TX 76798-7328, United States http://www.baylor.edu/math/

danieldsheng@gmail.com Westwood High School, Austin, TX 78750, United States http://schools.roundrockisd.org/Westwood/ 